Research Article

\title{
Further Results on a Curious Arithmetic Function
}

\author{
Long Chen, ${ }^{1}$ Kaimin Cheng $\mathbb{D},{ }^{2}$ and Tingting Wang ${ }^{3}$ \\ ${ }^{1}$ School of Mathematics and Computer Science, Panzhihua University, Panzhihua 617000, China \\ ${ }^{2}$ School of Mathematics and Information, China West Normal University, Nanchong 637002, China \\ ${ }^{3}$ Chengdu Experimental Foreign Language School, Chengdu 610061, China
}

Correspondence should be addressed to Kaimin Cheng; ckm20@126.com

Received 25 March 2020; Accepted 24 July 2020; Published 25 August 2020

Academic Editor: Efthymios G. Tsionas

Copyright (c) 2020 Long Chen et al. This is an open access article distributed under the Creative Commons Attribution License, which permits unrestricted use, distribution, and reproduction in any medium, provided the original work is properly cited.

Let $p$ be an odd prime number and $n$ be a positive integer. Let $v_{p}(n), \mathrm{N}^{*}$, and $\mathrm{Q}^{+}$denote the $p$-adic valuation of the integer $n$, the set of positive integers, and the set of positive rational numbers, respectively. In this paper, we introduce an arithmetic function $f_{p}: \mathrm{N}^{*} \longrightarrow \mathrm{Q}^{+}$defined by $f_{p}(n):=\left(n / p^{v_{p}(n)}\right)^{1-v_{p}(n)}$ for any positive integer $n$. We show several interesting arithmetic properties about that function and then use them to establish some curious results involving the $p$-adic valuation. Some of these results extend Farhi's results from the case of even prime to that of odd prime.

\section{Introduction}

Let $p$ be any given prime and $n$ be a positive integer. As usual, let $v_{p}(n), \mathrm{N}^{*}=\mathrm{N} \backslash\{0\}$, and $\mathrm{Q}^{+}=\{x \in \mathrm{Q} \mid x>0\}$ denote the $p$-adic valuation of the integer $n$, the set of positive integers, and the set of positive rational numbers, respectively. Arithmetic functions are classical topics in number theory (see, for example, [1-3]). Recent years, some kinds of periodic functions were introduced by some authors for the investigation of the least common multiple of integer sequences (see, for instance, [4-9]). In this paper, we introduce an interesting arithmetic function $f_{p}: \mathrm{N}^{*} \longrightarrow \mathrm{Q}^{+}$defined by

$$
f_{p}(n):=\left(\frac{n}{p^{v_{p}(n)}}\right)^{1-v_{p}(n)},
$$

for any positive integer $n$. We have, for example, $f_{5}(1)=$ $1, f_{5}(5)=1, f_{5}(50)=1 / 2, f_{5}(75)=1 / 3, f_{5}(250)=1 / 4$ etc. It is clear that $f_{p}(n)$ is not always an integer for any given prime $p$.

Throughout this paper, let $p$ always denote an odd prime. In this paper, we present several interesting arithmetic properties about that function and then use them to establish some curious results involving the $p$-adic valuation. This paper is organised as follows. First in Section 2, we show that the product $F_{p}(n):=\coprod_{r=1}^{n} f_{p}(r)$ is always an integer for any positive integer $n$, and it is, in fact, a multiple of all prime number not equal to $p$ and not exceeding $n$. Subsequently in Section 3, we first give a lower bound for $H_{p}(n)$ defined in Section 2. Also, two properties of $p$-adic valuation are provided. From these results, we can show that $\lim _{n \rightarrow \infty}$ $\left(F_{p}(n)\right)^{(1 / n)}$ does not exist for the odd prime $p$, which is very different from the case of $p=2$ since Farhi [10] proved that $\lim _{n \longrightarrow \infty}\left(F_{2}(n)\right)^{(1 / n)}=4$. Finally, Section 4 is devoted to derive an upper bound of $F_{p}(n)$ if $p \geq 3$. One notes that $f_{2}(n)$ was studied by Farhi [10], and $f_{3}(n)$ was investigated by Wang et al. [11]. Actually, many results they obtained would be extended in this paper to the case of all odd primes $p$.

In order to investigate the function $f_{p}$, two auxiliary arithmetic functions $g: \mathrm{Q}^{+} \longrightarrow \mathrm{N}^{*}$ and $h_{p}: \mathrm{N}^{*} \longrightarrow \mathrm{Q}^{+}$are needed, defined, respectively, by

$$
\begin{aligned}
& g(x):= \begin{cases}x, & \text { if } x \in \mathrm{N}^{*} \\
1, & \text { otherwise, }\end{cases} \\
& h_{p}(r):=\frac{r}{\prod_{i=1}^{\infty} g\left(r / p^{i}\right)},
\end{aligned}
$$

for any integer $r \in \mathrm{N}^{*}$. Note that the function $h_{p}$ is well defined since the product in the denominator of the right- 
hand side of (3) is actually finite because $g\left(r / p^{i}\right)=1$ for any sufficiently large $i$.

Let $x$ be a rational number. By $v_{p}(x)$, denote the $p$-adic valuation of $x$. Define $N_{p}(x)$ by

$$
N_{p}(x):=\frac{x}{p^{v_{p}(x)}} .
$$

So, $f_{p}(n)=N_{p}(n)^{1-v_{p}(n)}$ for any positive integer $n$. As usual, let $\lfloor\cdot\rfloor$ be the integer-part function, i.e., $\lfloor x\rfloor$ represents the largest integer no more than $x$, and $(\cdot \cdot)$ represent the binomial coefficient. Here, we give a well-known fact, which will be used frequently in this paper, as follows.

Fact 1. Each of following is true.

(i) $\lfloor(\lfloor x / a\rfloor) /(b)\rfloor=\lfloor x / a b\rfloor$ for any $a, b \in \mathrm{N}^{*}$ and $x \in \mathrm{Q}$.

(ii) (a) $e^{m} m !<(m+1)^{m+1}$.

(b) $\left(\begin{array}{c}((m+1) k) \\ k\end{array}\right)<\left(\left((m+1)^{m+1}\right) /\left(m^{m}\right)\right)^{k}$.

(c) $\left(\begin{array}{c}m \\ k\end{array}\right)<m^{k} / k$ ! hold for any positive integers $m$ and $k$.

(iii) (Legendre's formula) Let $p$ be a prime and $n$ be a positive integer. Then,

$$
v_{p}(n !)=\sum_{i=1}^{\infty}\left\lfloor\frac{n}{p^{i}}\right\rfloor=\frac{n-\sum_{i=0}^{s} a_{i}}{p-1}
$$

where $\sum_{i=0}^{s} a_{i} p^{i}$ is the $p$-adic representation of $n$.

\section{The Integrity of the Product Function}

Let $H_{p}(n):=\prod_{r=1}^{n} h_{p}(r)$ and $L_{n}:=\operatorname{lcm}(1, \ldots, n)$ for any positive integer $n$. Now, we give the first result as follows.

Theorem 1. Let $p$ be any given prime number and $n$ be a positive integer. Then, the value $F_{p}(n)$ is an integer.

Proof. First, by the definition of $g$, for any positive integer $r$, we have

$$
\begin{aligned}
\prod_{i=1}^{\infty} g\left(\frac{r}{p^{i}}\right) & =\prod_{i=1}^{\infty} g\left(p^{v_{p}(r)-i} N_{p}(r)\right) \\
& =\prod_{i=1}^{v_{p}(r)} g\left(p^{v_{p}(r)-i} N_{p}(r)\right) \\
& =\prod_{i=1}^{v_{p}(r)} p^{v_{p}(r)-i} N_{p}(r) \\
& =\prod_{i=0}^{v_{p}(r)-1} p^{i} N_{p}(r) \\
& =p^{\left(v_{p}(r)\left(v_{p}(r)-1\right) / 2\right)}\left(N_{p}(r)\right)^{v_{p}(r)} .
\end{aligned}
$$

Then,

$$
\begin{aligned}
h_{p}(r) & =\frac{r}{\prod_{i=1}^{\infty} g\left(r / p^{i}\right)}=p^{\left(v_{p}(r)\left(3-v_{p}(r)\right) / 2\right)}\left(N_{p}(r)\right)^{1-v_{p}(r)} \\
& =p^{\left(v_{p}(r)\left(3-v_{p}(r)\right) / 2\right)} f_{p}(r) .
\end{aligned}
$$

It follows that

$$
f_{p}(r)=p^{\left(v_{p}(r)\left(v_{p}(r)-3\right) / 2\right)} h_{p}(r) .
$$

So, for all positive integers $n$, we have

$$
F_{p}(n)=p^{\sum_{r=1}^{n}\left(v_{p}(r)\left(v_{p}(r)-3\right) / 2\right)} H_{p}(n) .
$$

Now, by applying the function $N_{p}$ into each of two sides of (9), we obtain

$$
\begin{aligned}
F_{p}(n) & =N_{p}\left(F_{p}(n)\right) \\
& =N_{p}\left(H_{p}(n)\right) .
\end{aligned}
$$

Therefore, to prove Theorem 1, we only need to prove that $H_{p}(n)$ is an integer for any positive integer $n$. For this purpose, by the definition of $g$, one notes that $\prod_{i=1}^{r} g(i / a)=$ $\lfloor r / a\rfloor$ ! for any positive integers $r$ and $a$. Hence,

$$
H_{p}(n)=\prod_{r=1}^{n} \frac{r}{\prod_{i=1}^{\infty} g\left(r / p^{i}\right)}=\frac{n !}{\prod_{i=1}^{\infty} \prod_{r=1}^{n} g\left(r / p^{i}\right)}=\frac{n !}{\prod_{i=1}^{\infty}\left\lfloor n / p^{i}\right\rfloor !}
$$

Additionally, $\quad \sum_{i=1}^{\infty}\left\lfloor n / p^{i}\right\rfloor \leq \sum_{i=1}^{\infty} n / p^{i}=n / p-1 \leq n$. Thus, $n ! /\left(\prod_{i=1}^{\infty}\left\lfloor n / p^{i}\right\rfloor !\right)$ is a multiple of the multinomial coefficient $\left(\begin{array}{c}\lfloor n / p\rfloor+\left\lfloor n / p^{2}\right\rfloor+\cdots \\ \lfloor n / p\rfloor,\left\lfloor n / p^{2}\right\rfloor, \ldots\end{array}\right)$ which is an integer. So, $n ! /\left(\prod_{i=1}^{\infty}\left\lfloor n / p^{i}\right\rfloor !\right)$ must be an integer. This finishes the proof of Theorem 1 .

Next, let us discuss the divisibility of $F_{p}(n)$ by $N_{p}\left(L_{n}\right)$. In fact, we have the following result.

Theorem 2. For any positive integer $n, F_{p}(n)$ is a multiple of $N_{p}\left(L_{n}\right)$. In particular, $F_{p}(n)$ is a multiple of all prime numbers not equal to $p$ and not exceeding $n$.

Proof. By (10) and (11), we know that to prove Theorem 2, it is sufficient to show that $n ! /\left(\prod_{i=1}^{\infty}\left\lfloor n / p^{i}\right\rfloor !\right)$ is a multiple of $L_{n}$, which is equivalent to prove that for all prime numbers $q$ not exceeding $n$, we have

$$
v_{q}\left(\frac{n !}{\prod_{i=1}^{\infty}\left\lfloor n / p^{i}\right\rfloor !}\right) \geq \alpha_{q}
$$

where $\alpha_{q}=v_{q}\left(L_{n}\right)$. On the one hand, by (i) and (iii) in Fact 1, one has 


$$
\begin{aligned}
v_{q}\left(\frac{n !}{\prod_{i=1}^{\infty}\left\lfloor n / p^{i}\right\rfloor !}\right) & =\sum_{i=1}^{\infty}\left\lfloor\frac{n}{q^{i}}\right\rfloor-\sum_{i=1}^{\infty} \sum_{j=1}^{\infty}\left\lfloor\frac{\left\lfloor n / q^{i}\right\rfloor}{p^{j}}\right\rfloor \\
& =\sum_{i=1}^{\infty}\left(\left\lfloor n / q^{i}\right\rfloor-\sum_{j=1}^{\infty}\left\lfloor\frac{\left\lfloor n / q^{i}\right\rfloor}{p^{j}}\right\rfloor\right) \\
& =\sum_{i=1}^{\alpha_{q}}\left(\left\lfloor\frac{n}{q^{i}}\right\rfloor-\sum_{j=1}^{\infty}\left\lfloor\frac{\left\lfloor n / q^{i}\right\rfloor}{p^{j}}\right\rfloor\right) .
\end{aligned}
$$

On the other hand, as $q \leq n$, for each $1 \leq i \leq \alpha_{q}$, one then gets

$$
\begin{aligned}
\left\lfloor\frac{n}{q^{i}}\right\rfloor-\sum_{j=1}^{\infty}\left\lfloor\frac{\left\lfloor n / q^{i}\right\rfloor}{\left.p^{j}\right\rfloor}\right. & \geq\left\lfloor\frac{n}{q^{i}}\right\rfloor-\sum_{j=1}^{\infty} \frac{\left\lfloor n / q^{i}\right\rfloor}{p^{j}} \\
& =\left\lfloor\frac{n}{q^{i}}\right\rfloor\left(1-\sum_{j=1}^{\infty} \frac{1}{p^{j}}\right) \\
& =\frac{p-2}{p-1}\left\lfloor\frac{n}{q^{i}}\right\rfloor>0 .
\end{aligned}
$$

Besides, $\left\lfloor n / q^{i}\right\rfloor-\sum_{j=1}^{\infty}\left\lfloor\left\lfloor n / q^{i}\right\rfloor / p^{j}\right\rfloor$ is an integer. It then infers that

$$
\left\lfloor\frac{n}{q^{i}}\right\rfloor-\sum_{j=1}^{\infty}\left\lfloor\frac{\left\lfloor n / q^{i}\right\rfloor}{p^{j}}\right\rfloor \geq 1 .
$$

Therefore by (13) and (15), we have

$$
v_{q}\left(\frac{n !}{\prod_{i=1}^{\infty}\left\lfloor n / p^{i}\right\rfloor !}\right) \geq \sum_{i=1}^{\alpha_{q}} 1=\alpha_{q},
$$

which implies that (12) is true. So, the proof of Theorem 2 is complete.

\section{Properties of $H_{p}(n)$ and $F_{p}(n)$}

Let $p$ be any odd prime. Now, we turn our attention to the lower bound for $H_{p}(n)$. In this section, we first obtain a lower bound for $H_{p}(n)$. Secondly, two properties of $p$-adic valuations will be given. Finally, one then concludes that $\lim _{n \longrightarrow \infty}\left(F_{p}(n)\right)^{(1 / n)}$ does not exist, which varies from the result of the case $p=2$ obtained by Farhi [10] that $\lim _{n \rightarrow \infty}\left(F_{2}(n)\right)^{(1 / n)}=4$. First of all, we present one lemma as follows.

Lemma 1. Let $p$ be a prime number. Let $m$ and $r$ be integers with $m \geq 1$ and $1 \leq r \leq p-1$. Then, for any positive integer $i$, one has

$$
\left\lfloor\frac{m p+r}{p^{i}}\right\rfloor=\left\lfloor\frac{m p}{p^{i}}\right\rfloor
$$

Proof. We prove (17) by induction on $i$ as follows.

For $i=1,(17)$ is clearly true.
For the given integer $i \geq 2$, suppose that (17) holds for any integer $j<i$. Let us now show that (17) is true for the integer $i$. On the one hand, by Fact 1, we have

$$
\left\lfloor\frac{m p+r}{p^{i}}\right\rfloor=\left\lfloor\frac{\left\lfloor m p+r / p^{i-1}\right\rfloor}{p}\right\rfloor .
$$

On the other hand, by the assumption, we have

$$
\left\lfloor\frac{m p+r}{p^{i-1}}\right\rfloor=\left\lfloor\frac{m p}{p^{i-1}}\right\rfloor .
$$

So, combining (18) with (19), it then follows from Fact 1 that

$$
\left\lfloor\frac{m p+r}{p^{i}}\right\rfloor=\left\lfloor\frac{m p}{p^{i}}\right\rfloor
$$

as desired. This completes the proof of Lemma 1.

Let $p \geq 3$ be a given prime number and $n$ be any positive integer. Now, we present a lower bound for $H_{p}(n)$.

Theorem 3. Let $p$ be an odd prime number and $n$ be a positive integer. Then, we have

$$
H_{p}(n) \geq\left(\log _{p} n\right)^{n} .
$$

Proof. Let $n$ be positive integer. First, we claim that

$$
H_{p_{1}}(n) \leq H_{p_{2}}(n),
$$

for all primes $p_{1}, p_{2}$ with $p_{1}<p_{2}$. In fact, for any positive integer $i$, one has

$$
\left\lfloor\frac{n}{p_{1}^{i}}\right\rfloor \geq\left\lfloor\frac{n}{p_{2}^{i}}\right\rfloor .
$$

It follows that

$$
\left\lfloor\frac{n}{p_{1}^{i}}\right\rfloor ! \geq\left\lfloor\frac{n}{p_{2}^{i}}\right\rfloor !,
$$

which infers that

$$
\prod_{i=1}^{\infty}\left\lfloor\frac{n}{p_{1}^{i}}\right\rfloor ! \geq \prod_{i=1}^{\infty}\left\lfloor\frac{n}{p_{2}^{i}}\right\rfloor !
$$

By (11), the desired result (22) follows. Now, let us continue to prove inequality (21). It is well known that $\log _{3} n \geq \log _{p} n$ for each positive integer $n$ and each prime $p \geq 3$. Hence, for proving inequality (21), by (22), we only need to prove

$$
H_{3}(n)=\frac{n !}{\prod_{i=1}^{\infty}\left\lfloor n / 3^{i}\right\rfloor !} \geq\left(\log _{3} n\right)^{n},
$$

which will be done in what follows.

By using a computer, we have checked that (26) is true for $1 \leq n \leq 3^{10}=59049$. Now, we show that (26) holds for any integer $n>3^{10}$ by induction on $n$. Let $n>3^{10}$ be the fixed positive integer. Assume that (26) is true for all integers less 
than $n$. We need to show that (26) is true for the integer $n$. We divide our proof into the following three cases.

Case 1. $n=3 m$, where $m$ is an integer with $m>3^{9}$. In this case, we have

$$
\begin{aligned}
H_{3}(n) & \left.=H_{3}(3 m)\right) \\
& =\frac{(3 m) !}{\left.\prod_{i=1}^{\infty} \mid 3 m / 3^{i}\right] !} \\
& =\frac{(3 m) !}{m ! \cdot m !} \cdot \frac{m !}{\left.\prod_{i=1}^{\infty} \mid m / 3^{i}\right] !} \\
& =\frac{(3 m) !}{m ! \cdot m !} H_{3}(m) .
\end{aligned}
$$

Then, by the assumption that $H_{3}(m) \geq\left(\log _{3} m\right)^{m} \geq 1$ and the facts that $(3 m) ! / m ! \cdot m ! \geq(2 m)^{m}\left(\begin{array}{c}2 m \\ m\end{array}\right) \geq(2 m)^{m}$, we have

$$
\begin{aligned}
H_{3}(n) \geq(2 m)^{m} & =\left(2 \times 3^{\log _{3} m}\right)^{m}>\left(\log _{3} m+1\right)^{3 m} \\
& =\left(\log _{3} 3 m\right)^{3 m} \\
& =\left(\log _{3} n\right)^{n},
\end{aligned}
$$

since $2 \times 3^{t}>(t+1)^{3}$ holds for any real number $t>9$. So, (26) is true if $3 \mid n$.

Case 2. $n=3 m+1$, where $m$ is an integer with $m \geq 3^{9}$. Then, by Lemma 1 and the assumption, we derive that

$$
\begin{aligned}
H_{3}(n) & =H_{3}(3 m+1) \\
& =\frac{(3 m+1) !}{\prod_{i=1}^{\infty}\left[3 m+1 / 3^{i}\right\rfloor !} \\
& =(3 m+1) \frac{(3 m) !}{\prod_{i=1}^{\infty}\left\lfloor 3 m / 3^{i}\right\rfloor !} \\
& =(3 m+1) H_{3}(3 m)>(3 m+1)\left(\log _{3} 3 m\right)^{3 m} .
\end{aligned}
$$

But one claims that

$$
\inf _{3 \leq t<+\infty} \frac{(t+1)\left(\log _{3} t\right)^{t}}{\left(\log _{3}(t+1)\right)^{t+1}}>1 .
$$

In fact, let $h(t):=\left((t+1)\left(\log _{3} t\right)^{t}\right) /\left(\left(\log _{3}(t+1)\right)^{t+1}\right)$ and $h^{\prime}(t)$ be the derivative function of $h(t)$. Then, one checks that $h \prime(t)>0$ for all real numbers $t \geq 3$. It follows that $h(t)$ increases monotonically over the interval $[3,+\infty)$. Then, $h(t) \geq h(3)=1.577669 \ldots$... So, (30) holds. Hence, from (29) and (30), we have that

$$
H_{3}(n)>\left(\log _{3}(3 m+1)\right)^{3 m+1}=\left(\log _{3} n\right)^{n} .
$$

This means that (26) is true if $3 \mid(n-1)$.

Case 3. $n=3 m+2$, where $m$ is an integer with $m \geq 3^{9}$. In this case, similar to Case 2, we have

$$
\begin{aligned}
H_{3}(n) & =(3 m+2) H_{3}(3 m+1)>\left(\log _{3}(3 m+2)\right)^{3 m+2} \\
& =\left(\log _{3} n\right)^{n} .
\end{aligned}
$$

This tells us that (26) holds if $3 \mid(n-2)$.

Combining the above cases, we have that (26) is true for the integer $n$. So, by mathematical induction, we have that (26) holds for any integer $n>3^{10}$. This completes the proof of Theorem 3.

In the following, we present two curious properties of $p$-adic valuations from $f_{p}(n)$

Theorem 4. Let $p$ and $q$ be distinct primes and $n$ be a positive integer. Then, we have

$$
\sum_{i=1}^{n} v_{p}(i) v_{q}(i) \leq \sum_{i=1}^{n} v_{q}(i)-\left\lfloor\log _{q} n\right\rfloor .
$$

Proof. On the one hand, by Theorem 2, we have

$$
\sum_{i=1}^{n} v_{q}\left(f_{p}(i)\right)=v_{q}\left(F_{p}(n)\right) \geq v_{q}\left(N_{p}\left(\left(L_{n}\right)\right)=v_{q}\left(L_{n}\right)=\left\lfloor\log _{q} n\right\rfloor .\right.
$$

On the other hand, for any integer $i \geq 1$, we get

$$
\begin{aligned}
v_{q}\left(f_{p}(i)\right) & =v_{q}\left(N_{p}(i)^{1-v_{p}(i)}\right) \\
& =\left(1-v_{p}(i)\right) v_{q}\left(N_{p}(i)\right) \\
& =\left(1-v_{p}(i)\right) v_{q}(i) .
\end{aligned}
$$

Therefore,

$$
\sum_{i=1}^{n}\left(1-v_{p}(i)\right) v_{q}(i) \geq\left\lfloor\log _{q} n\right\rfloor .
$$

So, the desired result follows. This finishes the proof of Theorem 4 .

Theorem 5. Let $p$ be a prime number and $n$ be a positive integer. Let $a_{0}+a_{1} p+a_{2} p^{2}+\cdots+a_{s} p^{s}$ be the $p$-adic expansion of $n$. Then, we have

$$
\sum_{i=1}^{n} v_{p}(i)\left(3-v_{p}(i)\right)=\frac{2 p-4}{(p-1)^{2}} \sum_{i=0}^{s}\left(p^{i}-1\right) a_{i}+\frac{2}{p-1} \sum_{i=0}^{s} i a_{i} .
$$

Proof. First, it is easy to see that (37) is true when $n<p$. So, in what follows, we let $n \geq p$. Then, (37) is equivalent to

$$
\sum_{i=1}^{n} v_{p}(i)\left(3-v_{p}(i)\right)=\frac{2 p-4}{(p-1)^{2}} \sum_{i=1}^{s}\left(p^{i}-1\right) a_{i}+\frac{2}{p-1} \sum_{i=1}^{s} i a_{i} \text {. }
$$

Now, we show that (38) holds. Taking the $p$-adic valuation on both sides of identity (9), using Fact 1 and (11), one has 


$$
\begin{aligned}
\sum_{i=1}^{n} \frac{v_{p}(i)\left(3-v_{p}(i)\right)}{2} & =v_{p}\left(H_{p}(n)\right) \\
& =v_{p}\left(\frac{n !}{\prod_{i=1}^{\infty}\left\lfloor n / p^{i}\right\rfloor !}\right) \\
& =\sum_{i=1}^{\infty}\left\lfloor\frac{n}{p^{i}}\right\rfloor-\sum_{i=1}^{\infty} \sum_{j=1}^{\infty}\left\lfloor\frac{n}{p^{i+j}}\right\rfloor
\end{aligned}
$$

One notes that $\#\left\{(i, j) \in \mathrm{N}^{* 2} \mid i+j=k\right\}=k-1$, where $k$ is an integer with $k \geq 2$. Then,

$$
\sum_{i=1}^{\infty} \sum_{j=1}^{\infty}\left\lfloor\frac{n}{p^{i+j}}\right\rfloor=\sum_{k=2}^{\infty} \sum_{i+j=k}^{\infty}\left\lfloor\frac{n}{p^{k}}\right\rfloor=\sum_{k=2}^{\infty}(k-1)\left\lfloor\frac{n}{p^{k}}\right\rfloor .
$$

$$
\sum_{i=1}^{n} \frac{v_{p}(i)\left(3-v_{p}(i)\right)}{2}=\sum_{i=1}^{\infty}\left\lfloor\frac{n}{p^{i}}\right\rfloor-\sum_{i=1}^{\infty} i\left\lfloor\frac{n}{p^{i+1}}\right\rfloor .
$$

Since

$$
(p-1) \sum_{i=1}^{\infty}\left((i-1)\left\lfloor\frac{n}{p^{i}}\right\rfloor-i\left\lfloor\frac{n}{p^{i+1}}\right\rfloor\right)=0,
$$

it then follows that

$$
\sum_{i=1}^{\infty}\left\lfloor\frac{n}{p^{i}}\right\rfloor-\sum_{i=1}^{\infty} i\left\lfloor\frac{n}{p^{i+1}}\right\rfloor=\sum_{i=1}^{\infty}\left(((p-1) i-p+2)\left\lfloor\frac{n}{p^{i}}\right\rfloor-\mathrm{pi}\left\lfloor\frac{n}{p^{i+1}}\right\rfloor\right) .
$$

However, by the $p$-adic expansion of $n: n=\sum_{i=j}^{s} a_{j} p^{j}$, one has

Then, (39) becomes

$$
((p-1) i-p+2)\left\lfloor\frac{n}{p^{i}}\right\rfloor-\mathrm{pi}\left\lfloor\frac{n}{p^{i+1}}\right\rfloor= \begin{cases}(p-2)(i-1)\left\lfloor\frac{n}{p^{i}}\right\rfloor+\mathrm{ia}_{i}, & \text { if } 1 \leq i \leq s \\ 0, & \text { if } i>s .\end{cases}
$$

Then, (43) together with (44) gives us that

$$
\sum_{i=1}^{\infty}\left\lfloor\frac{n}{p^{i}}\right\rfloor-\sum_{i=1}^{\infty} i\left\lfloor\frac{n}{p^{i+1}}\right\rfloor=\sum_{i=1}^{\infty}(p-2) i\left\lfloor\frac{n}{p^{i+1}}\right\rfloor+\sum_{i=1}^{s} \mathrm{ia}_{i},
$$

which infers that

$$
\sum_{i=1}^{\infty} i\left\lfloor\frac{n}{p^{i+1}}\right\rfloor=\frac{1}{p-1} \sum_{i=1}^{\infty}\left\lfloor\frac{n}{p^{i}}\right\rfloor-\frac{1}{p-1} \sum_{i=1}^{s} \mathrm{ia}_{i} .
$$

It then follows from (iii) of Fact 1 that

$$
\begin{aligned}
\sum_{i=1}^{\infty}\left\lfloor\frac{n}{p^{i}}\right\rfloor-\sum_{i=1}^{\infty} i\left\lfloor\frac{n}{p^{i+1}}\right\rfloor & =\frac{p-2}{p-1} \sum_{i=1}^{\infty}\left\lfloor\frac{n}{p^{i}}\right\rfloor+\frac{1}{p-1} \sum_{i=1}^{s} \mathrm{ia}_{i} \\
& =\frac{p-2}{(p-1)^{2}}\left(n-\sum_{i=1}^{s} a_{i}\right)+\frac{1}{p-1} \sum_{i=1}^{s} \mathrm{ia}_{i} \\
& =\frac{p-2}{(p-1)^{2}} \sum_{i=1}^{s}\left(p^{i}-1\right) a_{i}+\frac{1}{p-1} \sum_{i=1}^{s} \mathrm{ia}_{i} .
\end{aligned}
$$

Therefore, the desired result follows immediately from (41) and (47). The proof of Theorem 5 is complete.
Now, at the end of this section, by using Theorems 3 and 5 , one gets a result of limitation concerning $F_{p}(n)$ as follows.

Corollary 1. Let $p$ be an odd prime number. We have that

$$
\lim _{n \rightarrow \infty}\left(F_{p}(n)\right)^{(1 / n)}
$$

does not exist.

Proof. Let $p$ be an odd prime and $n$ be any positive integer. First, by (9) and Theorems 3 and 5, one has

$$
\begin{aligned}
F_{p}(n) & =p^{\sum_{r=1}^{n}\left(\left(v_{p}(r)\left(v_{p}(r)-3\right)\right) / 2\right)} H_{p}(n) \\
& =\frac{H_{p}(n)}{p^{\left((p-2) /(p-1)^{2}\right) \sum_{i=0}^{s}\left(p^{i}-1\right) a_{i}+(1 / p-1) \sum_{i=0}^{s} \mathrm{ia}_{i}}} \\
& \geq \frac{\left(\log _{p} n\right)^{n}}{p^{\left((p-2) /(p-1)^{2}\right) \sum_{i=0}^{s}\left(p^{i}-1\right) a_{i}+(1 / p-1) \sum_{i=0}^{s} \mathrm{ia}_{i}}},
\end{aligned}
$$

where $a_{0}+a_{1} p+a_{2} p^{2}+\cdots+a_{s} p^{s}$ is the $p$-adic representation of $n$. Now, taking $n=p^{m}$ with $m$ being a positive integer, one derives that 


$$
\begin{aligned}
\left(F_{p}(n)\right)^{(1 / n)}=\left(F_{p}\left(p^{m}\right)\right)^{\left(1 / p^{m}\right)} & \geq\left(\frac{\left(\log _{p} p^{m}\right)^{p^{m}}}{p^{\left((p-2) /(p-1)^{2}\right) p^{m}-\left((p-2) /(p-1)^{2}\right)+(m / p-1)}}\right)^{\left(1 / p^{m}\right)} \\
& =\frac{m}{p^{\left((p-2) /(p-1)^{2}\right)}\left(p^{-\left((p-2) /(p-1)^{2}\right)}\right)^{\left(1 / p^{m}\right)}\left(p^{(1 / p-1)}\right)\left(m / p^{m}\right)} \longrightarrow+\infty(m \longrightarrow+\infty)
\end{aligned}
$$

It then follows that

$$
\lim _{m \longrightarrow+\infty}\left(F_{p}\left(p^{m}\right)\right)^{\left(1 / p^{m}\right)}=+\infty .
$$

So, $\lim _{n \longrightarrow+\infty}\left(F_{p}(n)\right)^{(1 / n)}$ does not exist.

\section{An Upper Bound for $F_{p}(n)$}

Let $p$ be an odd prime. In this section, we give an upper bound for $F_{p}(n)$ as follows.

Theorem 6. Let $p$ be an odd prime number and $n$ be a positive integer. We have that

$$
F_{p}(n)<(\mathrm{pn})^{(p-2) n} 4^{n}
$$

Proof. First, we show that

$$
H_{p}(n)<(\mathrm{pn})^{(p-2) n} 4^{n}
$$

for all positive integers and odd primes $p$. This will be done by dividing into two cases.

Case 4. $n<p$. By the definition of $H_{p}(n)$ and (ii) of Fact 1 , one has

$$
H_{p}(n)=\prod_{r=1}^{n} h_{p}(r)=n !<\frac{(n+1)^{n+1}}{e^{n}}
$$

So, in order to prove (53) holds for any prime $p \geq 3$, we only need to confirm that

$$
\frac{(n+1)^{n+1}}{e^{n}} \leq(\mathrm{pn})^{(p-2) n} 4^{n}
$$

for $p \geq 3$ in the case of $n<p$, which will be easily done by taking logarithm into both sides of above inequality.

Case 5. $n \geq p$. Let us prove (53) by induction on $n$. First, it is easy to check (53) holds for $n=p$. Assume (53) is true for the integer less than $n$. Write $n=\mathrm{pm}+i$, where $m$ and $i$ are integers with $m \geq 1$ and $0 \leq i \leq p-1$. It then follows from (11) and Lemma 1 that

$$
\begin{aligned}
H_{p}(n) & =\frac{n !}{\prod_{r=1}^{\infty}\left\lfloor n / p^{r}\right\rfloor !} \\
& =\frac{(\mathrm{pm}+i) !}{\prod_{r=1}^{\infty}\left\lfloor(\mathrm{pm}+i) / p^{r}\right\rfloor !} \\
& =\frac{(\mathrm{pm}+i) !}{\prod_{r=1}^{\infty}\left\lfloor\mathrm{pm} / p^{r}\right\rfloor !} \\
& =((p-2) m+i) ! \times \frac{(\mathrm{pm}+i) !}{m ! m !((p-2) m+i) !} \frac{m !}{\prod_{r=1}^{\infty}\left\lfloor m / p^{r}\right\rfloor !} \\
& =((p-2) m+i) !\left(\begin{array}{c}
\mathrm{pm}+i \\
m, m,(p-2) m+i
\end{array}\right) H_{p}(m) \\
& =((p-2) m+i) !\left(\begin{array}{c}
2 m \\
\mathrm{pm}+i
\end{array}\right)\left(\begin{array}{c}
m \\
(p-2) m+i
\end{array}\right) H_{p}(m) .
\end{aligned}
$$

Hence, by utilizing inequalities in (ii) of Fact 1, one derives that

$$
\begin{aligned}
H_{p}(n) & <((p-2) m+i) ! 4^{m} \frac{(\mathrm{pm}+i)^{(p-2) m+i}}{((p-2) m+i) !} H_{p}(m) \\
& =4^{m}(\mathrm{pm}+i)^{(p-2) m+i} H_{p}(m) .
\end{aligned}
$$

So, (57) gives us from the assumption that

$$
\begin{aligned}
H_{p}(n) & <4^{m}(\mathrm{pm}+i)^{(p-2) m+i}(\mathrm{pm})^{(p-2) m} 4^{m}<4^{2 m} n^{2(p-2) m+i} \\
& <4^{n}(\mathrm{np})^{(p-2) n} .
\end{aligned}
$$

It follows that (53) holds for all integers $n$ with $n \geq p$. Therefore, (53) is proved. Now, by using (10) and (53), one arrives at

$$
F_{p}(n) \leq H_{p}(n)<(\mathrm{pn})^{(p-2) n} 4^{n},
$$

as desired. So, it completes the proof of Theorem 6 .

\section{Data Availability}

No data were used to support the study. 


\section{Conflicts of Interest}

The authors declare that they have no conflicts of interest.

\section{Authors' Contributions}

Kaimin Cheng provided the main idea of this paper and gave the proofs of the main theorems. Long Chen and Tingting Wang checked some details for the paper and did some work in writing this paper.

\section{Acknowledgments}

Kaimin Cheng would like to thank Professor Shuhong Gao for his warm hospitality and help during the former's visit to Clemson University in the year of 2020. Kaimin Cheng was supported partially by China Scholarship Council Foundation (201908510050) and the Research Initiation Fund for Young Teachers of China West Normal University (412679). Long Chen was supported partially by Doctoral Research Initiation Fund Project of Panzhihua University.

\section{References}

[1] T. M. Apostol, Introduction to Analytic Number Theory, Springer-Verlag, New York, NY, USA, 1976.

[2] G. H. Hardy and E. M. Wright, The Theory of Numbers, Oxford University Press, London, UK, Fifth edition, 1979.

[3] L. K. Hua, Introduction to Number Theory, Springer-Verlag, Berlin, Germany, 1982.

[4] B. Farhi, "Nontrivial lower bounds for the least common multiple of some finite sequences of integers," Journal of Number Theory, vol. 125, no. 2, pp. 393-411, 2007.

[5] B. Farhi and D. Kane, "New results on the least common multiple of consecutive integers," Proceedings of the American Mathematical Society, vol. 137, no. 6, pp. 1933-1939, 2009.

[6] S. Hong and G. Qian, "The least common multiple of consecutive arithmetic progression terms," Proceedings of the Edinburgh Mathematical Society, vol. 54, no. 2, pp. 431-441, 2011.

[7] S. Hong and Y. Yang, "On the periodicity of an arithmetical function," Comptes Rendus Mathematique, vol. 346, no. 13-14, pp. 717-721, 2008.

[8] S. Hong and G. Qian, "The least common multiple of consecutive quadratic progression terms," Forum of Mathematics, vol. 27, pp. 3335-3396, 2015.

[9] G. Qian, Q. Tan, and S. Hong, "On the periodicity of a class of arithmetic functions associated with multiplicative functions," Journal of Number Theory, vol. 133, no. 6, pp. 2005-2020, 2013.

[10] B. Farhi, "A study of a curious arithmetic function," Journal of Integer Sequences, vol. 15, no. 3, p. 9, 2012.

[11] T. Wang, Q. Tan, and S. Hong, "Arithmetic properties of a curious arithmetic function," in Proceedings of the International Conference on Algebra 2010, pp. 667-672, World Scientific Publishing, Hackensack, NJ, USA, December 2012. 